\title{
A Development Model of Semantic Web Application
}

\author{
Yinghui Huang, Guanyu Li and Xinlong Li \\ Faculty of Information Science and Technology, Dalian Maritime University, China \\ e-mail: hyh6599@163.com, rabitlee@163.com, lixinlonglxl@foxmail.com
}

Keywords: Semantic Web application; Development model; Application ontology; Knowledge base

\begin{abstract}
Semantic Web application is the application of semantic Web technology, of which the data containing semantic, thus is able to support the knowledge understanding and the knowledge processing. It can share information and refine information dynamically through the Internet access, which makes more full use of the existing Web resource on the Internet. In this paper, the definitions of semantic Web application as well as the structure and properties are formulated. A semantic Web development model is constructed with reference to the Web application development model.
\end{abstract}

\section{Introduction}

In order to solve the defects of Web in information express and information retrieval, Tim Berners-Lee [1], the father of Web, proposed the concept of semantic Web in 2001. At the same time, with the continuous evolution of semantic Web standards such as RDF, OWL and SPARQL in practical use, the exploration of semantic Web has begun towards the level of practical application from theoretical study and naturally semantic Web application has emerged.

Semantic Web application is developed with the help of semantic Web technology, its information is described by the concepts defined in domain ontology, namely the semantics which are given in the form of a standardized description. Semantic Web application makes it more fully to utilize the existing Web resources and exploit the implicit message in them. Although semantic Web technology has made many achievements in the fields of information express, information integration and information retrieval. The systematic description on the practice of semantic Web application based on semantic Web technology theory is rarely seen. Therefore, the discussion on the nature and the development model of semantic Web application is necessary.

Firstly, the definition, composition and properties of semantic Web application are given through the analysis of Web applications. Secondly, the functions of application ontology and knowledge base are discussed. Thirdly, a development model of semantic Web application is proposed and specific goals and implementation methods at every stage are explained detailedly. Finally, the obvious advantages on information integration, information share and information retrieval are verified by constructing a semantic Web application model.

\section{Semantic Web Application}

\section{Definition}

Semantic Web is called the third generation of Internet, which is aimed to handle and interpret Web information and is characterized by the interoperability on the semantic information among different machines. Semantic Web is the extension of the current Web, of which the information is marked by the concepts defined by domain ontology with rich semantics. Semantic Web expresses the semantics of the interrelated information through a standardized markup, which is unique and addressable. The markup makes it possible for the computer to understand and process information intelligently.

Web application is an application based on Web and is a product of typical B/S architecture. It is user-oriented and developed to complete a specific function, running on operating systems. The real core of Web application is to processing the data in distributed database. Web application also mean a computer software application that is coded in a browser-supported language (such as JavaScript) 
and reliant on a common web browser to render the application executable.

Semantic Web application [2-3] is an extension of Web application and also a product of B/S architecture. Semantic Web application is centered on the information marked by the concepts that are defined by application ontology, thus including rich semantics. It is concerned about taking full use of the existing diverse and distributed information on Web and aimed to achieve the purpose of sharing information through the semantic integration of the information. It supports dynamic and run-time data modification in order to adapt to current needs.

\section{Constitution}

Semantic Web application includes application ontology, knowledge base, knowledge base construction module, knowledge base inference module, knowledge base access module and results sorting module, as illustrated in Fig.1.

Application ontology is ontology targeted for specific areas, which consists of application ontology model, application ontology instance and application ontology rule. It provides a wealth of semantic support for the information of semantic Web application.

Knowledge base is transformed from the application ontology. It stores class, property and instance data defined by the application ontology.

Knowledge base construction module is

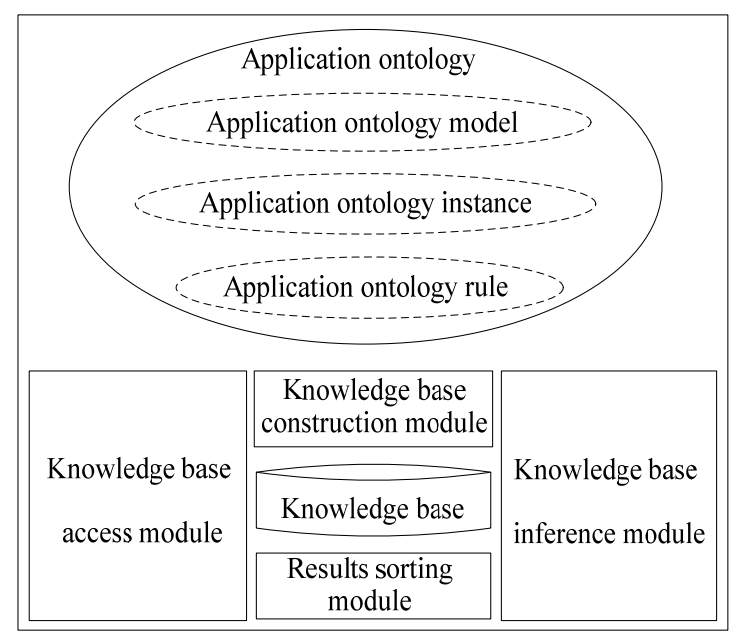

Figure 1. Semantic Web application model responsible for switching application ontology to knowledge base, mainly includes building the knowledge base, filling knowledge in it.

Knowledge base inference module is mainly responsible for knowledge inference by using some rules defined in the application ontology.

Knowledge base access module is mainly responsible for expressing a query request in the way of SPARQL [4], retrieving the knowledge base according to the inference results of the knowledge base, and then sending the retrieval results to the results sorting module.

Results sorting module is responsible for ranking the intensity of correlation between retrieval result and query request, then returning the sorted results to user.

The above is the introduction of the various modules, the following will describe the relationship between them.

As shown in Fig.1, application ontology is the basis for building semantic Web application, providing semantic support for the information in semantic Web application. Knowledge base is responsible for the information storage of semantic Web application. Knowledge base construction module transforms the concepts, the relationships between concepts in the application ontology into proper knowledge representation, and then stores them into the knowledge base. Knowledge base inference module and the knowledge base access module work around the knowledge base, responsible for completing the knowledge inference process based on knowledge base, as well as accessing to the knowledge base. Results sorting module sorts the retrieval results according to the size of relevance between the retrieval results and the retrieval requests, and then returns the sorted retrieval results to user.

\section{Properties}

Web data is the center of Semantic Web application. It takes advantage of the scale, diversity, and distribution found on the Web. The richness of semantic Web data lightens the programming burden. It decouples the data from programmed instructions and produces a cleaner, more flexible solution.

Semantic meaning is directly placed in the data of semantic Web application. It connects data through Web to improve the semantics and construct an expansive context for application.

Semantic Web can solve the conflicts between the semantics that relate similar concepts by using 
semantic Web statements. Therefore, the integration and sharing of data are more convenient in semantic Web application.

Semantic Web application allows the addition of new data at any time. This allows semantic Web application to gracefully align with current needs and avoids the traditional workarounds found in brittle designs [5].

\section{Development Model of Semantic Web Application}

\section{Development Model}

According to one of the mainstream software development methods [6], due to the components in object-oriented approach is constituted by class or object, therefore, a development model shown in Fig. 2 is proposed, which combined with the properties and characteristics of the semantic Web application. The development model mainly includes four phases: (1) object-oriented analysis, (2) object-oriented design, (3) object-oriented implementation and (4) object-oriented testing.

\section{Object-oriented Analysis}

The main task of the object-oriented analysis is to build object model, use case model and functional model of semantic Web application according to the problem description, which is similar to Web applications.

In addition, it is necessary to find the existing distributed Web data sources related to application backgrounds, basing on the background of issue, because semantic Web application is Web data-centric and designed to take full use
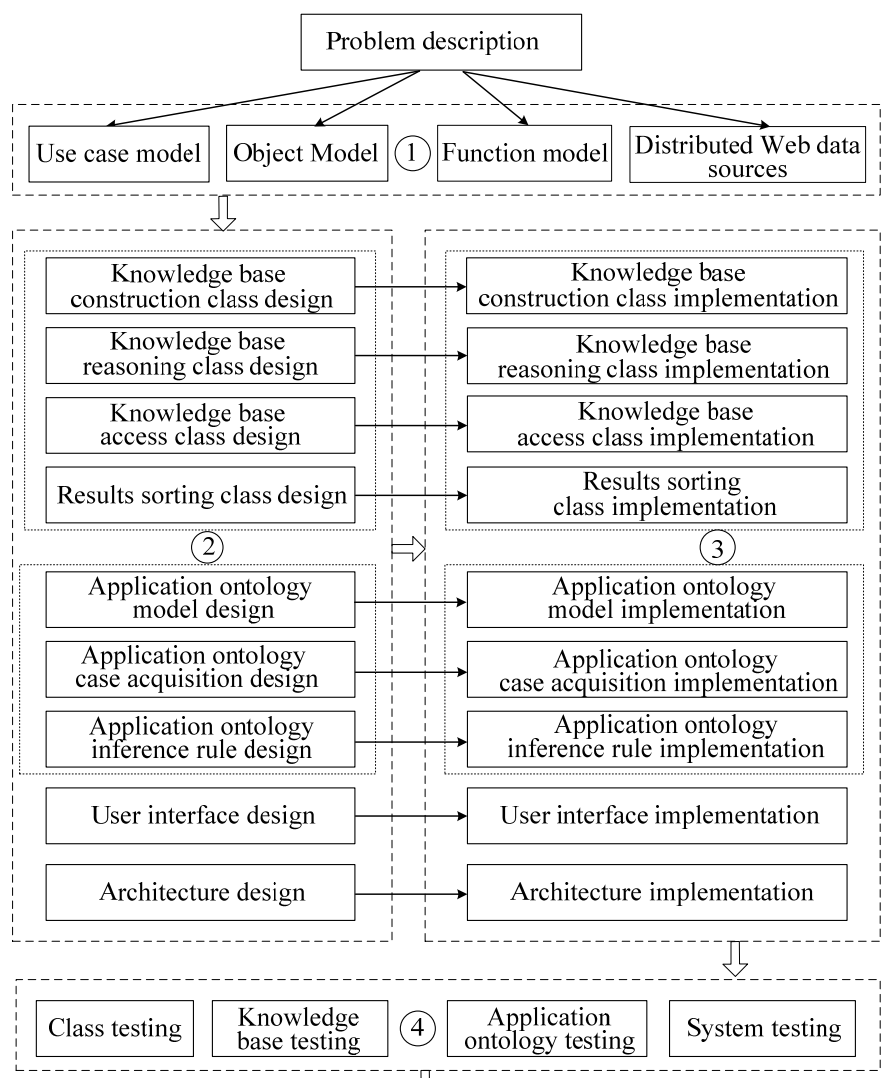

Semantic Web applications

Figure 2. Development model of semantic Web application of the diverse and distributed information on the Web, trying to avoid starting from scratch to create a data source.

\section{Object-oriented Design}

The main task of the object-oriented design is to process the model obtained in the object-oriented analysis phase further formally.

In semantic Web application, the main tasks of the design are software class design, application ontology design, user interface design and architecture design, among which the software class design includes knowledge base construction class design, knowledge base reasoning class design, knowledge base access class design and the results sorting class design that are reused and extended in other semantic Web applications. The obvious difference between semantic Web application and Web application is application ontology design.

Application ontology design consists of application ontology model design, application ontology case acquisition design and application ontology inference rule design.

Application ontology model design. Application ontology model refers to the model constructed by the relationship between concepts in the specific application backgrounds. It is achieved through the mutual mapping between object model and application ontology model. 
Application ontology model is built according to the model which is obtained in the object-oriented analysis phase. Application ontology is constituted by the relationship between concepts, constraint, instance, etc. Object model contains abstract class, objects, attributes and class hierarchy, corresponding to the major component of application ontology. The mapping relationship between the object model and the application ontology model is shown in Table 1.

Application ontology instance acquisition design. Since semantic Web application is Web data-centric, the purpose is to take full advantage of the diverse and distributed Web data [7]. The goal of the application ontology instance acquisition is to obtain the instance data of the application ontology by integrating the existing distributed data sources and to propose the model of the application ontology instance acquisition, as shown in Fig.3.

Data source ontologies in Fig.3 are expressed by the ontology description language RDF/OWL, corresponding to different ontologies of the existing data sources. First of all, use the ontology

Table 1. Object Model and Application Ontology Model

\begin{tabular}{cc}
\hline Object model & Application ontology model \\
\hline Class & Ontology class \\
Attribute & Object attribute / Data attribute \\
Class hierarchy & Ontology class hierarchy \\
\hline
\end{tabular}

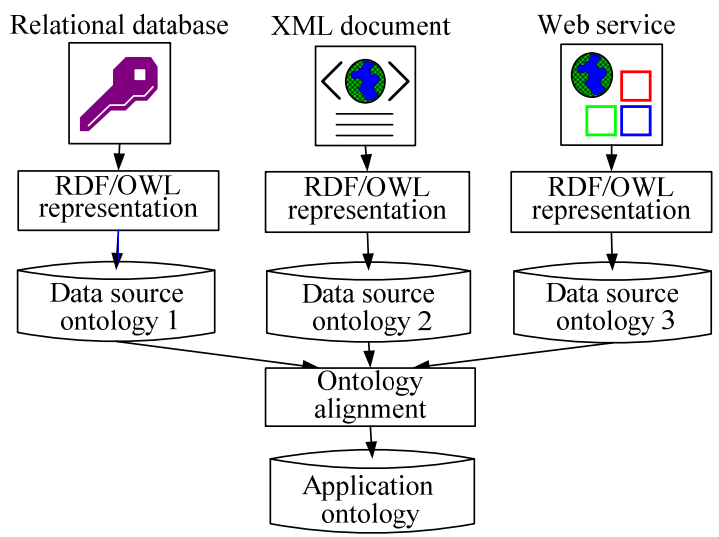

Figure 3. The model of application ontology instance acquisition description language RDF/OWL to describe different types of the existing data sources and then get data source ontology for respective data source. Secondly, create the mapping relationship between the data source ontology and the application ontology, using ontology alignment technique. Finally, convert the instance data of source ontology into the instance data of application ontology.

Application ontology inference rule design. Rule is one of the ways to represent knowledge and increase the ability to express knowledge. The rules in semantic Web application are in the form of if-then clause. Choose the common SWRL [8] rules and the Jena [9] rules specifically. It is not only to improve the knowledge expression ability but also to make knowledge-based inference possible, using concepts, relationships between the concepts, instances and relations between instances in application ontology to define inference rules.

\section{Object-oriented Implementation}

Object-oriented implementation is to use the object-oriented programming language to design various components of the model physically, including the realization of objects' methods and properties, connections between objects and user interface, etc. In semantic Web application, the main tasks in the object-oriented implementation phase are to implement software class, application ontology, user interface and architecture.

In the object-oriented implementation phase of semantic Web application, some existing semantic Web frameworks, such as the Jena framework and the RDF framework [10] are made use of, which normally consists of three basic components: storage component (knowledge base), access component (query processing program) and inference component (inference engine). The class of each component is realized fully. As the Jena framework is open source and the most popular in the present, it can meet the needs of semantic Web application development. Furthermore, as one of the main forms of semantic Web application is semantic retrieval, so for a semantic Web application, the main task in the object-oriented implementation phase is focus on how to achieve semantic retrieval. Therefore, this paper chooses the Jena framework to achieve the semantic retrieval in semantic Web application.

The semantic retrieval model based on the Jena framework is shown in Fig.4. 


\section{Object-oriented Testing}

As semantic Web application has its own characteristics, not only class testing and system testing but also application ontology testing and knowledge base testing should be done in the testing phase.

Application ontology testing mainly refers to the testing for mode, instance and rule. For example, the parent-child relationship in the mode is complete or not, the domain and ranges are full or not, the definition of the instance class is complete or not and the inference of the defined rules is effective or not.

Knowledge base testing mainly refers to the performance testing for knowledge base. The most commonly used indicators are query duration and load time while other indicators are such as inference level supported, inference correctness and delete duration, etc.

As semantic Web application is an extension of Web application, so it should obtain B/S architecture through four steps: object-oriented analysis, object-oriented design, object-oriented implementation and object-oriented testing.

\section{Summary}

This paper describes the definition, composition and nature of semantic Web application in detail. The development model of semantic Web application is given. It is easily to build semantic Web applications through the development model of semantic Web application which can applied to many practical aspects such as semantic retrieval.

Semantic Web application, constructed by the development model of semantic Web application, reflects the data-centric perspective of semantic Web data and achieves the information integration of distributed Web data sources. The use of inference rules improves presentation skills of information.

\section{Acknowledgment}

This paper is supported by the National Natural Science Foundation of China under grant No. 60972090, "Research on Imprecision Model of Semantic Web Ontology”.

\section{References}

[1] T. Berners-Lee, J. Hendler, and O. Lassila, The semantic Web, Scientific American, 2001, vol. 5: pp. 34-43.

[2] W.M. Fitzgerald, S.N. Foley, Aligning semantic Web applications with network access controls, Computer Standards \& Interfaces, 2011, vol. 33(1): pp. 24-34.

[3] C. Omer, S. Henry, Semantic eye: a semantic Web application to rationalize and enhance chemical electronic publishing, J. Chem. Inf. Model., 2007, vol. 46(6): pp. 2396-2411.

[4] W3C, SPARQL query language for RDF, http://www.w3.org/TR/rdf-sparql-query/, 15 January, 2008.

[5] J. Hebeler, M. Fisher, R. Blace, and et al, Semantic Web programming, Wiley publishing, Inc, Indianapolis, 2009.

[6] E. Oren, R. Delbru, S. Gerke, and et al, ActiveRDF: object-oriented semantic Web programming, Proceedings of ACM, 2007, pp. 817-824.

[7] C.C. Lo, Integrating semantic Web and object-oriented programming for cooperative design, Journal of Universal Computer Science, 2009, vol. 15(9): pp. 1970-1990.

[8] W3C, SWRL: A semantic Web rule language combining OWL and RuleML, http://www.w3.org/Submission/SWRL/, 21 May, 2004. 
[9] B. McBride, Jena: a semantic Web toolkit, Internet Computing, 2002, vol. 6(6): pp. 55-59.

[10]B.V. Aduna, User guide for Sesame 2.3, http://www.openrdf.org/doc/sesame2/2.3.2/users/ index.html, 16 July, 2010.

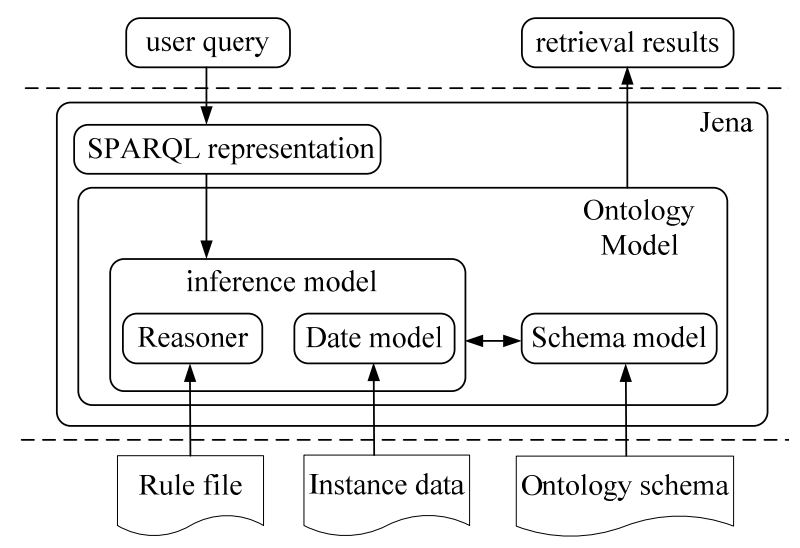

Figure 4. Semantic retrieval model based on the Jena framework 